\title{
Volume of Distribution of Fraction Dose Observed Normalized by Dose
}

National Cancer Institute

\section{Source}

National Cancer Institute. Volume of Distribution of Fraction Dose Observed Normalized by Dose. NCl Thesaurus. Code C102729.

The volume of distribution associated with the terminal slope following extravascular administration divided by the fraction of dose absorbed, calculated using the observed value of the last non-zero concentration, divided by the dose. 